\title{
Early cellular responses of BMSCs genetically modified with bFGF/BMP2 co-cultured with ligament fibroblasts in a three-dimensional model in vitro
}

\author{
BIN LI $^{1 *}$, RAMESH KUMAR JHA ${ }^{1 *}$, YONG-JIAN QI ${ }^{1}$, QU-BO NI ${ }^{1}$, \\ HUI WANG $^{2}$, BIAO CHEN ${ }^{1}$ and LIAO-BIN CHEN ${ }^{1}$ \\ ${ }^{1}$ Department of Orthopaedic Surgery, Zhongnan Hospital, Wuhan University; \\ ${ }^{2}$ Department of Pharmacology, Basic Medical School, Wuhan University, Wuhan, P.R. China
}

Received January 1, 2016; Accepted September 7, 2016

DOI: 10.3892/ijmm.2016.2752

\begin{abstract}
Currently, a number of strategies including the implantation of bone marrow-derived mesenchymal stem cells (BMSCs) and growth factors have been developed to regenerate the tendon-to-bone interface after performing anterior cruciate ligament reconstruction. However, the mechanisms behind the interactions of the implanted BMSCs and tendon cells remain to be elucidated. The aim of this study was to evaluate the early cellular responses of BMSCs genetically modified with basic growth factor growth factor (bFGF)/bone morphogenic protein 2 (BMP2) and ligament fibroblasts in a three-dimensional co-culture model. BMSCs and ligament fibroblasts were both isolated from male Wistar rats. The BMSCs were then transfected with an adenoviral vector carrying bFGF or BMP2. The transfected BMSCs and ligament fibroblasts both encapsulated in alginate beads were co-cultured for 6 days in three-dimensional model. On days 0,3 and 6 , cell proliferation was assayed. On day 6 , the expression of several tendon-bone related markers was evaluated. In the co-culture system, bFGF and BMP2 were highly expressed at the mRNA and protein level. During the process, bFGF significantly promoted cell proliferation, as well as the expression of scleraxis (SCX) and collagen (COL) type I (COL1) in the BMSCs; however, it markedly decreased the expression of phenotype markers in the ligament fibroblasts, including COL1 and COL3. BMP2 markedly increased the expression of alkaline phosphatase and osteocalcin in the BMSCs and ligament fibroblasts, whereas it had no obvious effect on cell proliferation and collagen synthesis
\end{abstract}

Correspondence to: Dr Biao Chen or Professor Liao-Bin Chen, Department of Orthopaedic Surgery, Zhongnan Hospital, Wuhan University, Donghu Road 169, Wuhan, Hubei 430071, P.R. China

E-mail: chenbiao20030701@163.com

E-mail: lbchen@whu.edu.cn

*Contributed equally

Key words: bone marrow-derived mesenchymal stem cells, ligament fibroblasts, basic fibroblast growth factor, bone morphogenic protein 2, transfection, co-culture in the ligament fibroblasts. The combination of bFGF and BMP2 resulted in the similarly enhanced proliferation of BMSCs and ligament fibroblasts as observed with bFGF alone; however, this combination more potently promoted osteogenic differentiation than did BMP2 alone. The findings of our study demonstrate the superiority of the combined use of growth factors in inducing osteogenic differentiation and provide a theoretical foundation for the regeneration of the tendon-to-bone interface.

\section{Introduction}

The anterior cruciate ligament (ACL) plays a pivotal role in maintaining the stability of the knee (1). The normal structural composition of the native ACL-to-bone interface consists of four distinct tissue regions, including the ligament, the non-mineralized fibrocartilage, mineralized fibrocartilage and bone. Due to the relative avascularity of the fibrocartilage zone and bone loss at the site of injury (2), the anatomic insertion site is difficult to regenerate, which has led orthopedic surgeons to perform ACL reconstructions in the majority of cases (3). Functional rehabilitation post-ACL reconstruction largely depends on the successful healing of the tendon-to-bone interface (4).

In recent years, a number of strategies, including cell therapy and various growth factors in tissue engineering have been investigated in an aim to biologically accelerate and improve the healing of tendon-to-bone interface $(5,6)$. Bone marrowderived mesenchymal stem cells (BMSCs) have been utilized to investigate the regeneration of the tendon-to-bone interface due to their self-renewal potential and pluripotency for possible clinical use (7-11). However, there is still unsatisfactory rehabilitation resulting from the lack of adequate growth factors which have been shown to be powerful regulators of biological function (12). Bone morphogenetic protein 2 (BMP2) belongs to the transforming growth factor (TGF) $\beta$ superfamily, known for its osteoinductive capacity, which has been well-investigated in the study of tendon-to-bone healing $(13,14)$. Dong et al reported that the topical application of BMSCs infected with recombinant BMP2 lentivirus promoted the formation of fibrocartilage-like tissue and further improved the mechanical properties of the reconstructed ACL (15), indicating that the interactions between cells derived from BMSCs and tendon 
cells (fibroblasts) play a role in fibrocartilage formation by initiating the differentiation of BMSCs and phenotypic alteratoins in fibroblasts through suitable growth factors.

Basic fibroblast growth factor (bFGF) has been found to be involved in numerous cellular functions, including angiogenesis, cell proliferation, wound healing, limb formation and tissue remodeling (16-19). However, to date, there are only a few studies available on the effects of bFGF (by gene therapy) on tendon-to-bone regeneration following ACL reconstruction. Kohno et al demonstrated the abundance of bFGF and BMP2 at the tendon-bone interface through an immunohistochemical investigation during the early post-operative stage (12). Their results implied that the implanted BMSCs genetically modified with both bFGF and BMP2 may potentially promote tendon-tobone regeneration.

To examine this hypothesis, we designed a three-dimensional BMSC-ligament fibroblast co-culture model using an alginate hydrogel microsphere, which mimics the cellular organization at the interface in vivo, as well as facilitating paracrine interactions. To the best of our knowledge, this is the first report of the interaction of gene-transfected BMSCs with ACL-derived fibroblasts. We also evaluated the early effects of co-culture on the differentiation of BMSCs and the phenotypic maintenance of ligament fibroblasts. The findings of our study provide a theoretical foundation for the regeneration of the tendon-to-bone interface.

\section{Materials and methods}

Construction of adenoviral vectors. Replication-defective human adenovirus type 5 (SinoGenoMax Co., Ltd., Beijing, China) was used to generate the recombinant adenoviral vectors as previously described (20). To obtain adenoviral vectors carrying bFGF and BMP2, the human entire coding sequence of bFGF (480 bp) and BMP2 (1.2 kb) was inserted into an adenoviral plasmid containing an enhanced green fluorescent protein (EGFP) under the control of the cytomegalovirus (CMV) promoter (SinoGenoMax Co., Ltd.). The produced vectors were designated as AdbFGF and AdBMP2. Subsequently, 293 cells purchased from the American Type Culture Collection (ATCC, Manassas, VA, USA) were used to reproduce recombinant viruses, as previously described (21). Viral titers were measured using a fluorescence spectrophotometer (F-4500; Hitachi, Tokyo, Japan), as previously described (22). The 50\% tissue culture infective dose $\left(\mathrm{TCID}_{50} / \mathrm{ml}\right)$ was utilized to detect the quantification of virus infectious titers, as previously described (23).

Isolation, expansion and characterization of BMSCs. Two specific pathogen-free Wistar rats weighing 80-100 g (male) were purchased from the Laboratory Animal Center of Wuhan University (Wuhan, China). The protocol for the use of rats was approved by the Committee on the Ethics of Animal Experiments of Wuhan University. The animals were sacrificed by anesthesia with $5 \%$ isoflurane. The femurs and tibias were harvested and the metaphysis on both sides was removed using a rongeur under sterile conditions. Bone marrow was collected by flushing the femur and tibia with medium. Following centrifugation at $150 \mathrm{x} \mathrm{g}$ for $8 \mathrm{~min}$ at $25^{\circ} \mathrm{C}$, the cell pellets were mixed thoroughly with Dulbecco's modified Eagle's medium (DMEM)/F12 (Invitrogen, Carlsbad, CA, USA) supplemented with $10 \%$ fetal bovine serum (Gibco, Grand Island, NY, USA) and $100 \mathrm{U} / \mathrm{ml}$ penicillin-streptomycin in $5 \% \mathrm{CO}_{2}$ at $37^{\circ} \mathrm{C}$ and the cells were subcultured to passage 3 for the following experiments. The pluripotency of the BMSCs was confirmed by culturing for osteogenic and chondrogenic differentiation in controlled medium as previously described $(24,25)$. Generally, for osteogenic differentiation, the BMSCs were seeded at a density of $4 \times 10^{4}$ cells/ $\mathrm{cm}^{2}$ in a 6-well plate for 2 weeks in the osteogenic medium and von Kossa staining (Baso Biotech Co., Ltd., Wuhan, China) was then utilized to detect the calcium deposits. For chondrogenic differentiation, the BMSCs were suspended at a concentration of $5 \times 10^{6}$ cells $/ \mathrm{ml}$ in $1.25 \%$ alginate (Sigma-Aldrich, St. Louis, MO, USA) in $0.15 \mathrm{M} \mathrm{NaCl}$ and slowly dropped into $102 \mathrm{mM} \mathrm{CaCl}_{2}$ solution and then encapsulated in alginate beads. After 4 weeks of culture, the alginate bead sections were stained with Alcian blue (ALB; Baso Biotech Co., Ltd.) and Safranin O (Saf-O; Baso Biotech Co., Ltd.) for the evaluation of chondrogenesis. Furthermore, BMSC markers were also analyzed by flow cytometry. Approximately $5 \times 10^{5}$ cells were incubated with specific phycoerythrin- or fluorescein isothiocyanate-conjugated monoclonal antibodies for rat CD29 (25-0291), CD90 (15-0900), CD45 (11-0461) and CD11 (12-0110) (Biolegend, San Diego, CA, USA) and subjected to flow cytometric analysis using a BD FACSAria ${ }^{\mathrm{TM}}$ III flow cytometer (BD Biosciences, San Jose, CA, USA).

Isolation and expansion of ligament fibroblasts. The tissue of ACLs were removed from both knee joints of the rats under aseptic conditions and sliced into sections (approximately $1 \mathrm{~mm}^{3}$ ) and then digested with $0.2 \%$ collagenase type I (Sigma, Santa Clara, CA, USA) for $3 \mathrm{~h}$ at $37^{\circ} \mathrm{C}$ in an incubator. Following centrifugation at $150 \mathrm{xg}$ for $8 \mathrm{~min}$ at $25^{\circ} \mathrm{C}$, the supernatant was discarded, and the recovered cells were cultured with the same growth medium described above and subcultured to passage 3 for use in the following experiments.

Gene transfer and establishment of three-dimensional co-culture model. Adenoviral vectors were transfected into the BMSCs at a multiplicity of infection (MOI) of 0, 25, 50, 100, 150 and 200. After $48 \mathrm{~h}$, the cells were harvested and used to detect EGFP expression by flow cytometry, and the cells were also observed under an inverted fluorescence microscope (4J41302; Olympus, Tokoyo, Japan). The percentage of live infected cells expressing EGFP was counted to determine the optimal MOI value. Each experiment was repeated at least 3 times. Additionally, the expression of the transfected genes was examined by reverse transcription-quantitative polymerase chain reaction (RT-qPCR).

The BMSCs were transfected with AdEGFP, AdbFGF, or AdBMP2, or with AdbFGF plus AdBMP2. At 24 h posttransfection, the BMSCs were trypsinized and encapsulated in the beads following the method described above. Twenty beads were placed on one side of the well each containing a sterile satinless metal cell strainer. The third passage ligament fibroblasts cultured in alginate beads at a concentration of $5 \times 10^{6}$ cells $/ \mathrm{ml}$ were placed on the other side of the well. Based on the different treatment of the BMSCs, the co-culture model was divided into 4 groups as follows: i) the AdEGFP group, ii) the AdbFGF group, iii) the AdBMP2 group, iv) the AdbFGF plus AdBMP2 group. The co-cultured cells were incubated for 6 days and the medium was changed every 3 days in all groups. 
Table I. Sequences of primers for used for RT-qPCR.

\begin{tabular}{|c|c|c|}
\hline Genes & Primer sequences & $\begin{array}{l}\text { Annealing } \\
\text { temp }\left({ }^{\circ} \mathrm{C}\right)\end{array}$ \\
\hline GAPDH & $\begin{array}{l}\text { F: GCAAGTTCAACGGCACAG } \\
\text { R:GCCAGTAGACTCCACGACA }\end{array}$ & 60 \\
\hline bFGF & $\begin{array}{l}\text { F: GTGTTACGGATGAGTGTTTCT } \\
\text { R:CAGCTCTTAGCAGACATTGG }\end{array}$ & 60 \\
\hline BMP2 & $\begin{array}{l}\text { F: AGTGGGTGCTGCTCTTCCTA } \\
\text { R:ATGGGACACTCCTCTGTTGG }\end{array}$ & 60 \\
\hline SCX & $\begin{array}{l}\text { F: TGGGTGAAGCCTGCGGTGAC } \\
\text { R:CGTCTTTCTGTCACGGTCTTTGCT }\end{array}$ & 60 \\
\hline $\mathrm{OCN}$ & $\begin{array}{l}\text { F: CAGACCTAGCAGACACCATG } \\
\text { R:GCTTGGACATGAAGGCTTTG }\end{array}$ & 60 \\
\hline ALP & $\begin{array}{l}\text { F: GCCTTACCAACTCATTTGTGC } \\
\text { R:CATACCATCTCCCAGGAACATG }\end{array}$ & 60 \\
\hline COL1 & $\begin{array}{l}\text { F: CATGTCTGGTTTGGAGAGAG } \\
\text { R:CGCTGTTCTTGCAGTGATA }\end{array}$ & 60 \\
\hline COL3 & $\begin{array}{l}\text { F: CTGGAGTCGGAGGAATGG } \\
\text { R:GCCAGATGGACCAATAGCA }\end{array}$ & 60 \\
\hline
\end{tabular}

PCR, polymerase chain reaction; GAPDH, glyceraldehydes-3-phosphate dehydrogenase; bFGF, basic growth factor growth factor; BMP2, bone morphogenic protein 2; SCX, scleraxis; OCN, osteocalcin; ALP, alkaline phosphatase; COL1, collagen type I; COL3, collagen type III; F, forward; R, reverse.

Cell proliferation assay. The proliferation of the transfected BMSCs and the ligament fibroblasts in the co-culture model was determined on days 0,3 and 6. Briefly, on days 0,3 and 6 , the tests were performed on 96-well plates with one bead in each well. Culture medium containing MTS solution (Promega, Shanghai, China) was added followed by incubation for $4 \mathrm{~h}$ at $37^{\circ} \mathrm{C}$, as previously described (26). The absorbance at $490 \mathrm{~nm}$ was then measured using a spectrophotometer (Shimadzu, Kyoto, Japan).

Concentration of $b F G F$ and BMP2 in the cell supernatant. The concentrations of bFGF and BMP2 in the cell supernatant following co-culture for 3 days were determined using enzyme linked immunosorbent assay (ELISA) kits (R\&D Systems, Minneapolis, MN, USA) according to the manufacturer's instructions.

$R N A$ extraction and $R T-q P C R$. mRNA expression of related genes in the BMSCs and ligament fibroblasts was detected by RT-qPCR. Following co-culture for 6 days, the BMSCs and ligament fibroblasts were recovered from the alginate beads. Total RNA was isolated using TRIzol reagent (Invitrogen) following manufacturer's instructions, and was converted to cDNA using the PrimScript ${ }^{\circledR}$ RT reagent kit (Takara Biotechnology Co., Ltd., Dalian, China). cDNA was assayed for mRNA expression, including that of glyceraldehyde-3-phosphate dehydrogenase (GAPDH), scleraxis (SCX), collagen (COL) type 1 (COL1), alkaline phosphatase (ALP), osteocalcin (OCN) and COL3. The tests were performed on an ABI Step One RT-PCR thermal cycler (ABI Stepone, Applied Biosystems, Foster City, CA, USA) using the SYBR ${ }^{\circledR}$ Premix Ex Taq ${ }^{\mathrm{TM}}$ kit (Takara Biotechnology Co., Ltd.). The housekeeping gene, GAPDH, was used as a quantitative control. The sequences of the primers used for PCR and the annealing temperature for the genes used in this experiment are shown in Table I. The PCR cycling conditions were as follows: pre-denaturation at $95^{\circ} \mathrm{C}$ for $30 \mathrm{sec}$, denaturation at $95^{\circ} \mathrm{C}$ for $5 \mathrm{sec}$, and the annealing conditions for each gene, and final elongation at $72^{\circ} \mathrm{C}$ for $30 \mathrm{sec}$. The $\mathrm{Ct}$ value of fluorescent product was detected at the extension period, and gene expression in all samples was analyzed by applying the $2^{-\Delta \Delta \mathrm{Ct}}$ relative quantification method.

Western blot analysis. Proteins were extracted from the harvested BMSCs and ligament fibroblasts on day 6 following co-culture. The protein concentrations were determined by BCA assay (Sigma). Samples of $50 \mu \mathrm{l}$ protein were separated on $12 \%$ sodium dodecyl sulphate-polyacrylamide gels before being transferred onto polyvinylidene difluoride membranes (Millipore, Boston, MA, USA). The membranes were blocked with $5 \%$ non-fat milk powder and incubated with anti-SCX (1:250; sc-87425), anti-ALP (1:200; sc-79839), anti-OCN (1:1,000; sc-18319), anti-COL1 (1:100; sc-8784) and anti-COL3 (1:150; sc-8781) antibodies (all from Santa Cruz Biotechnology Co., Ltd., Santa Cruz, CA, USA), and then incubated with peroxidase-conjugated secondary antibodies (A0001H; Bluegene Biotech Co., Ltd., Shanghai, China). The proteins were visualized by ECL detection (Amersham Pharmacia Biotech, Piscataway, NJ, USA) following the manufacturer's instructions. GAPDH (sc-48166; Santa Cruz Biotechnology Co., Ltd.) was used as an internal control.

Statistical analysis. For the quantification of data, each assay was repeated at least 3 times independently. The results of the quantitative analyses are expressed as the means \pm standard error of mean (SEM). The means were compared using one-way analysis of variance (one-way ANOVA) followed by Tukey's post-hoc assuming equal variances with the SPSS 17 (SPSS, Inc., Chicago, IL, USA). A value of $\mathrm{P}<0.05$ was considered to indicate a statistically significant difference.

\section{Results}

Characterization of BMSCs. Firstly, BMSCs exhibited a long spindle-like shape and grew well under the microscope (Fig. 1A). Subsequently, to identify whether the isolated cells were rat BMSCs, the pluripotency of the BMSCs was assessed by differentiation toward the osteogenic lineage by von Kossa staining (Fig. 1B) and the chondrogenic lineage by ALB staining (Fig. 1C) and Saf-O staining (Fig. 1D). Black calcium deposits were spotted and the alginate beads sections were positively stained for glycosaminoglycan with ALB and Saf-O staining, which indicated the pluripotency of the BMSCs. Lastly, we performed flow cytometric analysis to demonstrate that the rat BMSCs expressed the specific mesenchymal stem cell markers, CD29 and CD90, but not the hematopoietic lineage markers, CD11 and CD45 (Fig. 1E).

Efficiency of adenoviral infection of BMSCs and successful establishment of the co-culture model. The infection efficiency firmly correlated with the dosage of adenoviral vectors. The efficiency was $89.1 \%$ at an MOI of 50 and $95.5 \%$ of the 

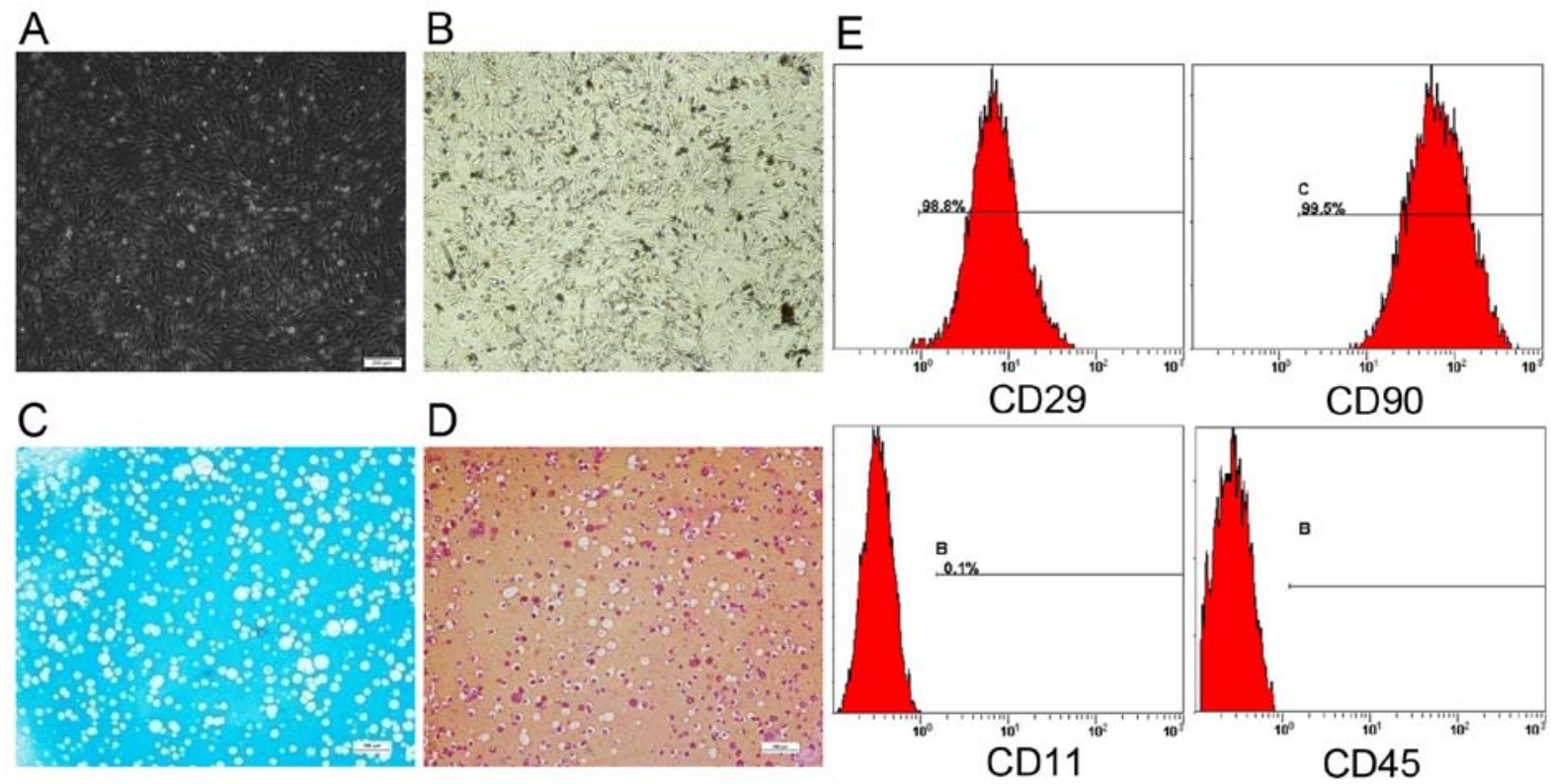

Figure 1. Characterization of rat bone marrow-derived mesenchymal stem cells (BMSCs). (A) The morphology of BMSCs was photographed under a phase-contrast microscope. (B) BMSCs were stained with von Kossa staining following osteogenic differentiation. BMSCs in alginate beads were stained with (C) Alcian blue (ALB) and (D) Safranin O (Saf-O) following chondrogenic differentiation. Scale bar, $100 \mu \mathrm{m}$. (E) Flow cytometric analysis of expression of the BMSCs markers (CD29 and CD90) and hematopoietic markers (CD11 and CD45).

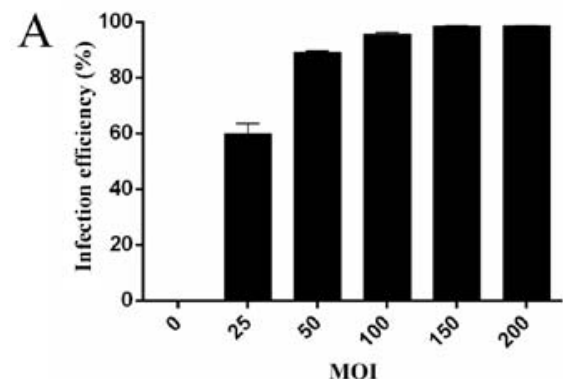

$\mathrm{B}$

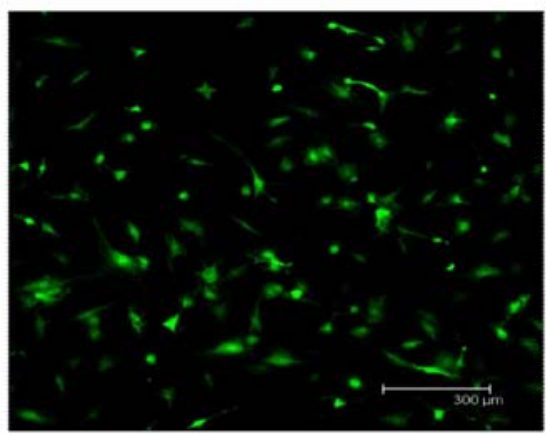

$\mathrm{C}$

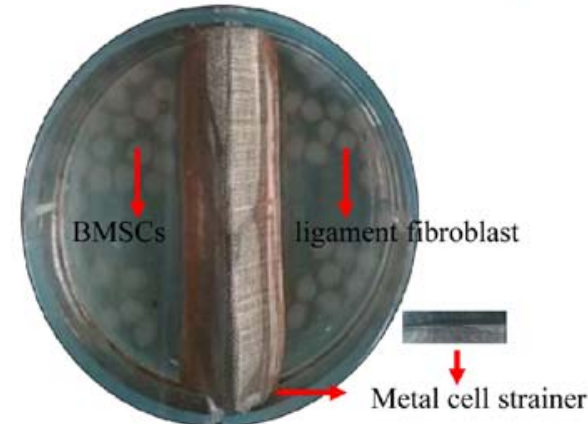

D

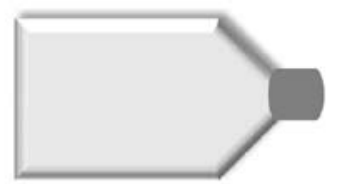

BMSCs

!

Adenovirus

transfection

Alginate/cell

suspension

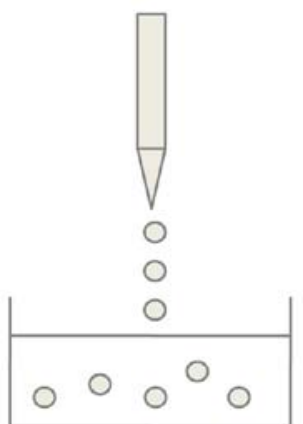

$\downarrow$ Metal cell strainer $\downarrow$

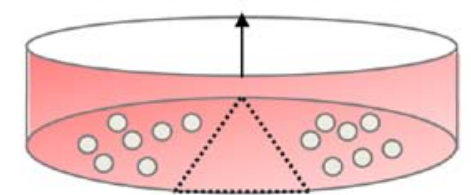

Figure 2. Determination of adenoviral infection efficiency and establishment of co-culture model. (A) Quantitative assessment of transfection efficiency was performed by flow cytometric analysis. (B) Photomicrograph of transfected bone marrow-derived mesenchymal stem cells (BMSCs) showing the GFP-positive cells at an MOI of 50 visualized under a fluorescence microscope. Scale bar, $300 \mu \mathrm{m}$. (C and D) Presentation of co-culture model of transfected BMSCs and ligament fibroblasts both encapsulated in alginate beads. 

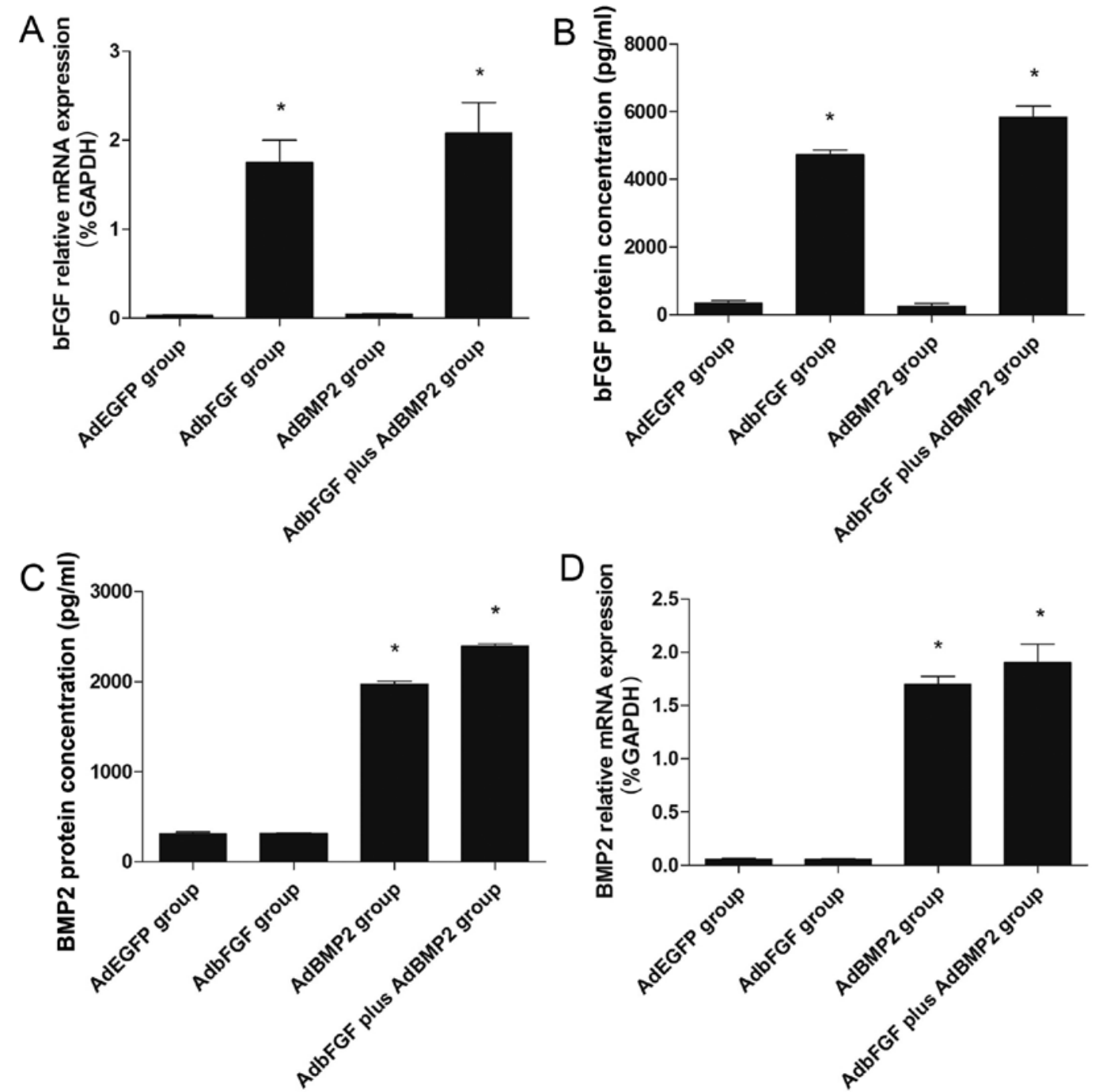

Figure 3. mRNA and protein expression of basic growth factor growth factor (bFGF) and bone morphogenic protein 2 (BMP2) in the supernatant of bone marrow-derived mesenchymal stem cells (BMSCs) transfected with adenovirus. mRNA expression of (A) bFGF and (D) BMP2 in BMSCs within $72 \mathrm{~h}$ of transfection analyzed by RT-qPCR. Following co-culture for 6 days, the protein concentration of (B) bFGF and (C) BMP2 was determined by ELISA. Bars represent the means \pm SEM. ${ }^{*} \mathrm{P}<0.05$ compared with the AdEGFP group.

BMSCs were infected at an MOI of 100 (Fig. 2A). The basic principle to determine the optimal MOI is selecting the most cost-effective recombinant virus with the least cytotoxicity and higher infection efficiency; we selected the MOI of 50 as the optimal MOI. Fluorescence microscopic visualization revealed a high efficiency of adenovirus at an MOI 50 following transfection (Fig. 2B). The co-culture model developed by our own laboratory was established using a stainless metal cell strainer in a 6-well plate (Fig. 2C and D).

Gene and protein expression of $b F G F$ and $B M P 2$. The gene expression of bFGF and BMP2 within $72 \mathrm{~h}$ following transfection was verified by RT-qPCR. The mRNA expression of bFGF was markedly increased in the AdbFGF group and in the AdbFGF plus AdBMP2 group, compared with the AdEGFP group (Fig. 3A). The mRNA expression of BMP2 was significantly enhanced in the AdBMP2 group and in the AdbFGF plus AdBMP2 group, compared with the AdEGFP group (Fig. 3D).

Similar to the increase observed in mRNA expression, the protein concentrations in the supernatant were also increased. The protein concentration of bFGF was higher in the AdbFGF group (4731.57 $\pm 224.82 \mathrm{pg} / \mathrm{ml})$ and in the AdbFGF plus AdBMP2 group $(5835.27 \pm 568.27 \mathrm{pg} / \mathrm{ml})$, than in the AdEGFP group (343.17 $\pm 134.18 \mathrm{pg} / \mathrm{ml})$ following co-culture for 3 days (Fig. 3B). The protein concentration of BMP2 was higher in the Ad-BMP2 group $(1948.47 \pm 326.86 \mathrm{pg} / \mathrm{ml})$ and in the AdbFGF plus AdBMP2 group $(2135.33 \pm 228.09 \mathrm{pg} / \mathrm{ml})$, than in the AdEGFP group $(312.16 \pm 31.85 \mathrm{pg} / \mathrm{ml})$ following co-culture for 3 days (Fig. 3C).

Cell proliferation assay. BMSC proliferation exhibited no obvious changes among the groups on day 0 , which suggested the non-toxicity of the adenovirus on BMSC viability following transfection at the indicated MOI. Cell proliferation was markedly enhanced in the AdbFGF group and in the AdbFGF plus AdBMP2 group on days 3 and 6. However, there was no significant difference between the AdBMP2 group and AdEGFP group (Fig. 4A).

Moreover, ligament fibroblast proliferation exhibited similar changes to those of the BMSCs. Cell proliferation was 


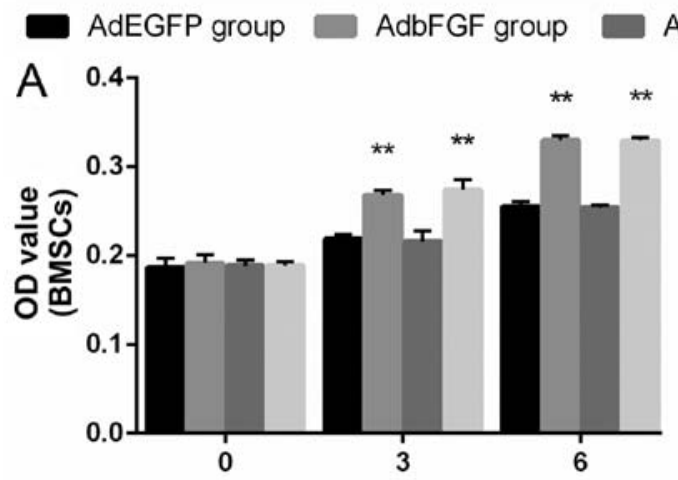

AdBMP2 group AdbFGF plus AdBMP2 group

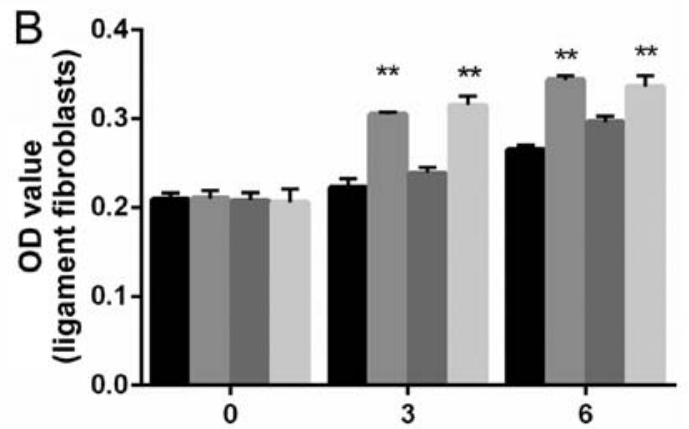

Figure 4. Analysis of the proliferation of bone marrow-derived mesenchymal stem cells (BMSCs) and ligament fibroblasts by MTS assay. BMSCs were cocultured with ligament fibroblasts and the proliferation of (A) BMSCs and (B) ligament fibroblasts was then determined by MTS assay on days 0,3 and 6. Bars represent the means $\pm \mathrm{SEM} .{ }^{* * *} \mathrm{P}<0.01$ compared with the AdEGFP group.
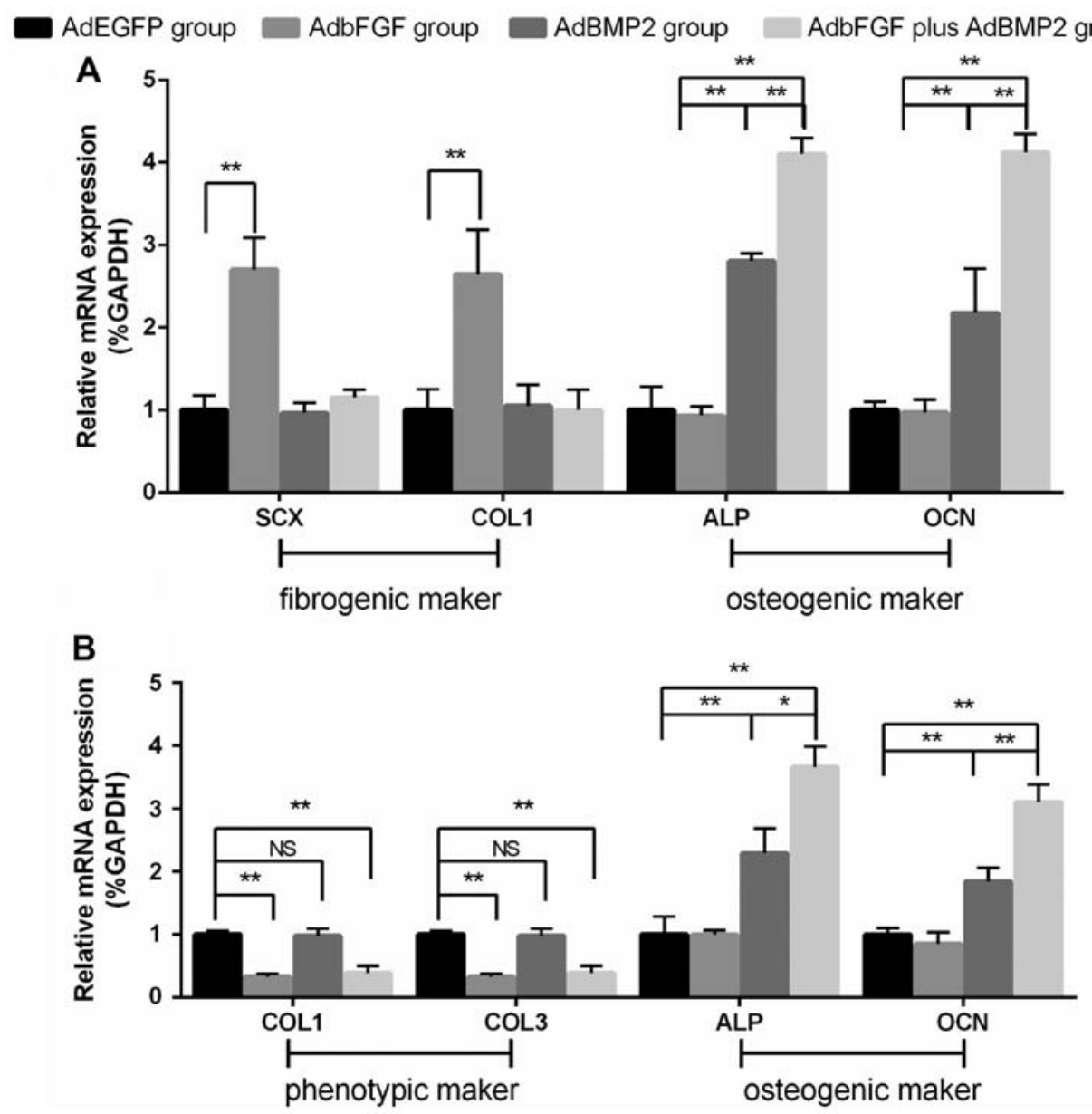

Figure 5. mRNA expression of bone marrow-derived mesenchymal stem cells (BMSCs) and ligament fibroblasts determined by RT-qPCR. The mRNA expression of scleraxis (SCX), collagen (COL) type I (COL1), alkaline phosphatase (ALP) and osteocalcin (OCN) in (A) BMSCs and that of COL1, COL3, ALP and OCN in (B) ligament fibroblasts was analyzed by RT-qPCR following co-culture for 6 days. Bars represent the means \pm SEM. ${ }^{*} \mathrm{P}<0.05$ and ${ }^{* * *} \mathrm{P}<0.01$ compared with the AdEGFP group. NS, not significant.

distinctly increased in the AdbFGF group and in the AdbFGF plus AdBMP2 group on days 3 and 6; however, no significant difference was observed between the AdBMP2 group and AdEGFP group (Fig. 4B).

$m R N A$ and protein expression in cells by RT-qPCR and western blot analysis. As regards the BMSCs (Figs. 5A and 6A), compared with the AdEGFP group, the mRNA and protein expression of SCX and COL1 was significantly elevated in the AdbFGF group. In addition, the mRNA and protein expression of ALP and OCN was distinctly increased in the AdBMP2 group and in the AdbFGF plus AdBMP2 group. As regards the ligament fibroblasts (Figs. 5B and 6B), compared with the AdEGFP group, the mRNA and protein expression of COL1 

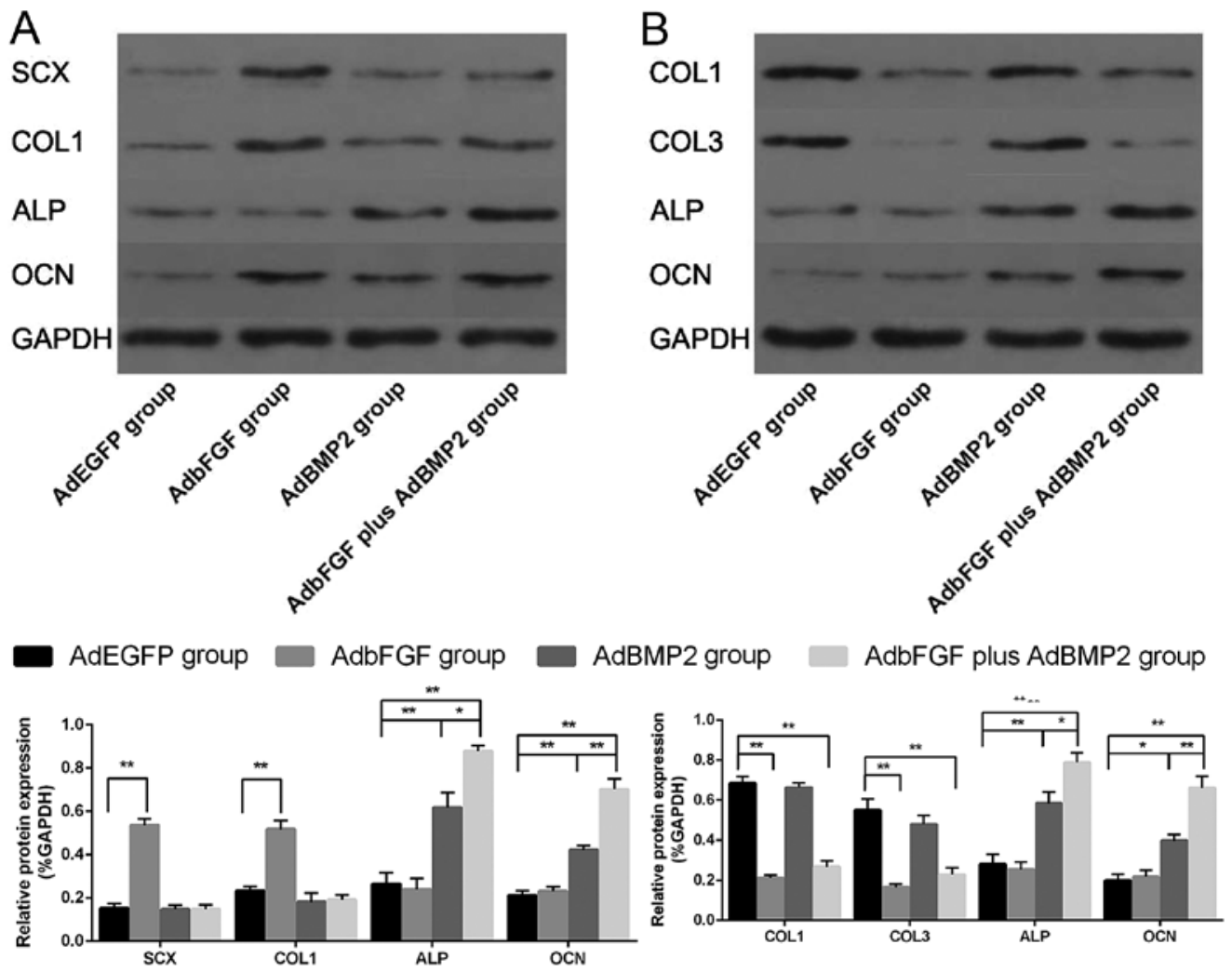

Figure 6. Protein levels in bone marrow-derive3d mesenchymal stem cells (BMSCs) and ligament fibroblasts confirmed by western blot analysis following co-culture for 6 days. The protein expression of scleraxis (SCX), collagen type I (COL1), alkaline phosphatase (ALP) and osteocalcin (OCN) in (A) BMSCs and that of COL1, COL3, ALP and OCN in (B) ligament fibroblasts was detected by western blot analysis following co-culture for 6 days. GAPDH was used as an internal control. ${ }^{*} \mathrm{P}<0.05$ and ${ }^{* *} \mathrm{P}<0.01$ compared with the AdEGFP group.

and COL3 was significantly decreased in the AdbFGF group and in the AdbFGF plus AdBMP2 group, although there was no obvious difference between the AdBMP2 group and the AdEGFP group. Moreover, the mRNA and protein expression of ALP and OCN was markedly enhanced in the AdBMP2 group and AdbFGF plus AdBMP2 group. In addition, a higher expression of ALP and OCN in both the BMSCs and ligament fibroblasts was observed in the AdbFGF plus AdBMP2 group compared with the AdBMP2 group.

\section{Discussion}

Our goal was to elucidate the possible mechanisms responsible for the regeneration of the tendon-to-bone interface, and this study focused on the early cellular interactions between BMSCs expressing bFGF/BMP2 and ligament fibroblasts. A biomimetic three-dimensional co-culture model was developed to evaluate the interactions between transfected BMSCs and ligament fibroblasts and to analyze the potential effects of these communications on the interface. Our results revealed that the combination of $\mathrm{bFGF}$ and BMP2 promoted the proliferation and osteogenic differentiation of the BMSCs. On the other hand, the two cytokines not only promoted the proliferation and differentiation of the ligament fibroblasts, but also decreased the expression of COL1 and COL3, which are the main components of the ligament matrix. These effects in the co-culture system suggest that the implantation of BMSCs expressing bFGF/BMP2 at the interface may achieve a solid osseointegration between the grafts and the bone tunnel.
Scaffolds are important components of the tissue engineering strategy as they define the ultimate shape of the construct while providing the required mechanical strength during regeneration and proper cell attachment sites (27). The alginate hydrogel microsphere as a unique three-dimensional cell delivery scaffold, has been widely used in tissue engineering, drug delivery and wound healing, due to retainment of the structural similarity with the extracellular tissues (28). Due to these features, we adopted this strategy to encapsulate both genetically modified BMSCs that served as a source of growth factors and ligament fibroblasts for maintaining the phenotype during culture (29). It has been confirmed that macromolecules with molecular weight of $<49 \mathrm{kDa}$ can penetrate the pores of the alginate hydrogel microspheres to influence cell behavior (30), which suggessts that $\mathrm{bFGF}$ and BMP2 can enter the microsphere and regulate the biological behavior of encapsulated BMSCs and ligament fibroblasts. Furthermore, our previous study also reported the proliferation and differentiation of BMSCs in alginate hydrogel microspheres for cartilage tissue engineering (25). Thus, it is possible for alginate hydrogel microspheres to be used as scaffolds in tissue engineering techniques.

SCX, detected in the ligament progenitor cells, is important for the development of the musculoskeletal system and COL1 and COL 3 is the major constituent of ligament $(31,32)$. In our study, for the AdbFGF group, our results demonstrated that cell proliferation and the expression of SCX and COL1 in the BMSCs was markedly enhanced, indicating a shift of BMSCs towards the more mature state of fibroblast-like cells following the gene transfer of bFGF. Our findings collaborate with the findings of 
the in vitro model by Cai et al, which suggested that bFGF may be an important regulator of the proliferation and differentiation of BMSCs (33). As regards the ligament fibroblasts, cell proliferation was markedly enhanced, and this was accompanied by the decreased expression of COL1 and COL3. Qiu et al reported similar results, showing that the expansion of tenocytes treated by bFGF was supported, while collagen synthesis was significantly decreased (34). Caliari and Harley also confirmed that bFGF increased the proliferation of equine tenocytes, but reduced the expression of phenotype-related genes (COL1 and COL3) within an anisotropic collagen-GAG scaffold, for which they considered that a single factor led to a dose-dependent trade-off between driving tenocyte proliferation versus the maintenance of a tenocyte phenotype (31). In this study, the possible reason may be that for the expansion phase of fibroblasts in vitro, early cell differentiation and target structure formation has to be minimized to enhance nutrient diffusion. Fully differentiated fibroblasts tend to form thick layers of collagen around the scaffold, which could prevent the cells within the scaffold from gaining sufficient nutrients from the culture medium and consequently would be less likely to proliferate.

In this study, with respect to the AdBMP2 group, the early osteogenic differentiation of the BMSCs was noted, evidenced by the increased expression of OCN and ALP, which was consistent with other findings reported by other studies $(35,36)$. However, the proliferation and the expression of collagen (COL1 and COL3) concerning the exposure of ligament fibroblasts to BMP2 exhibited no obvious changes. Other in vitro studies have reported consistent results that neither the proliferation of tenocyte-like cells nor collagen production was influenced by BMP2 $(13,37,38)$. In our study, the increased expression of OCN and ALP in ligament fibroblasts was also observed. This result supports those of the study by Salingcarnboriboon et al, who showed that the mRNA expression of ALP and osterix in tendon cell lines (TT-E4, TT-G11 and TT-D6) was extensively increased following culture in the presence of BMP2 for 3 days (38). Additionally, Steinert et al demonstrated that ACL-derived cells express stem cell markers and are able to undergo osteogenic differentiation (39). Our present results indicated that BMP2 promoted the osteogenic differentiation of ligament fibroblasts. Hashimoto et al reported that an engineered bone-to-bone graft, generated by injecting BMP2 into the semitendinosus tendon to achieve ectopic ossicles, resulted in the restoration of morphology and function equivalent to those of the normal ACL (40). Martinek et al reported that adenoviral BMP-2 transfection of ACL grafts led to improved bone tunnel integration in rabbits (41). The effects of osteogenic differentiation of BMP2 on the ligament fibroblasts in our findings may be a reason for the improved tendon-to-bone healing as reported above and further suggest that a beneficial effect of implanted BMSCs expressing BMP2 may be partially caused by enhancing the osteogenic differentiation of both BMSCs and ligament fibroblasts.

A cocktail of various growth factors has been developed to manipulate the biological healing of the tendon-to-bone interface. Hou et al revealed that the healing of experimentally injured Achilles tendons in rabbits would be enhanced by the cell-based gene transfer of vascular endothelial growth factor (VEGF) and TGF- $\beta 1$ (42). In an in vitro study, Pauly et al investigated BMP-2 in combination with BMP-7 and showed that it could positively affect human rotator cuff ligament fibroblasts in terms of stimulating cell activity and COL1 production and the expression of several markers (13). In this study, the combination of bFGF and BMP2 yielded better results, since the combination of both factors more potently promoted the proliferation and osteogenic differentiation of BMSCs and ligament fibroblasts along with the decreased expression of collagen in the ligament fibroblasts, suggesting the synergistic effects of bFGF and BMP2 in the co-culture system. Wang et al demonstrated similar results, showing that the combination of BMP-2 and bFGF was more effective than either one alone in promoting the formation of new bone (43). Based on the findings of this study, it may be presumed that the synergistic effects firstly began with the continuous fission and proliferation of BMSCs and ligament fibroblasts, as well as the decreased expression of phenotype makers in ligament fibroblasts induced by bFGF. During this process, BMP2 was provided to induce cell differentiation. However, the detailed mechanisms responsible for these synergistic effects and whether more benefits could be achieved if more genes are transfected warrants further investigation.

The duration (6 days) was simply provided for our preliminary knowledge about the early cellular responses of BMSCs transfected with bFGF/BMP2 and ligament fibroblast in threedimensional co-culture. However, as a limitation to our study, the terminal effects of long term co-culture between the two types of cells in vitro remain unknown. In addition, further studies are required to confirm whether the co-application of bFGF and BMP2 will achieve a solid osseointegration between the grafts and the bone tunnel in vivo.

In conclusion, we developed a biomimetic three-dimensional co-culture model to evaluate the interactions between transfected BMSCs and ligament fibroblasts. Co-culture of two types of cells gave rise to cell differentiation and phenotypic changes. The findings of this study demonstrated the superiority of combinational growth factors in inducing osteogenic differentiation and provided a theoretical foundation for the improvement of the tendon-to-bone interface in vivo.

\section{Acknowledgements}

This study was supported by grants from the Natural Science Foundation of China (no. 81201401 and 81371940).

\section{References}

1. Laurencin CT and Freeman JW: Ligament tissue engineering: An evolutionary materials science approach. Biomaterials 26 : 7530-7536, 2005

2. Wong MW, Qin L, Tai JK, Lee SK, Leung KS and Chan KM: Engineered allogeneic chondrocyte pellet for reconstruction of fibrocartilage zone at bone-tendon junction - a preliminary histological observation. J Biomed Mater Res B Appl Biomater 70: 362-367, 2004.

3. Zantop T, Petersen W, Sekiya JK, Musahl V and Fu FH: Anterior cruciate ligament anatomy and function relating to anatomical reconstruction. Knee Surg Sports Traumatol Arthrosc 14: 982-992, 2006.

4. Kurosaka M, Yoshiya S and Andrish JT: A biomechanical comparison of different surgical techniques of graft fixation in anterior cruciate ligament reconstruction. Am J Sports Med 15: 225-229, 1987.

5. Rothrauff BB and Tuan RS: Cellular therapy in bone-tendon interface regeneration. Organogenesis 10: 13-28, 2014. 
6. Atesok K, Fu FH, Wolf MR, Ochi M, Jazrawi LM, Doral MN, Lubowitz JH and Rodeo SA: Augmentation of tendon-to-bone healing. J Bone Joint Surg Am 96: 513-521, 2014.

7. Li YG, Wei JN, Lu J, Wu XT and Teng GJ: Labeling and tracing of bone marrow mesenchymal stem cells for tendon-to-bone tunne healing. Knee Surg Sports Traumatol Arthrosc 19: 2153-2158, 2011.

8. Ouyang HW, Goh JC and Lee EH: Use of bone marrow stromal cells for tendon graft-to-bone healing: Histological and immunohistochemical studies in a rabbit model. Am J Sports Med 32: 321-327, 2004

9. Gulotta LV, Kovacevic D, Ehteshami JR, Dagher E, Packer JD and Rodeo SA: Application of bone marrow-derived mesenchymal stem cells in a rotator cuff repair model. Am J Sports Med 37: 2126-2133, 2009.

10. Gulotta LV, Kovacevic D, Montgomery S, Ehteshami JR, Packer JD and Rodeo SA: Stem cells genetically modified with the developmental gene MT1-MMP improve regeneration of the supraspinatus tendon-to-bone insertion site. Am J Sports Med 38: 1429-1437, 2010

11. Lim JK, Hui J,Li L, Thambyah A, Goh J and Lee EH: Enhancement of tendon graft osteointegration using mesenchymal stem cells in a rabbit model of anterior cruciate ligament reconstruction. Arthroscopy 20: 899-910, 2004.

12. Kohno T, Ishibashi Y, Tsuda E, Kusumi T, Tanaka M and Toh S: Immunohistochemical demonstration of growth factors at the tendon-bone interface in anterior cruciate ligament reconstruction using a rabbit model. J Orthop Sci 12: 67-73, 2007.

13. Pauly S, Klatte F, Strobel C, Schmidmaier G, Greiner S Scheibel M and Wildemann B: BMP-2 and BMP-7 affect human rotator cuff tendon cells in vitro. J Shoulder Elbow Surg 21: 464-473, 2012

14. Wang CJ, Weng LH, Hsu SL, Sun YC, Yang YJ, Chan YS and Yang YL: pCMV-BMP-2-transfected cell-mediated gene therapy in anterior cruciate ligament reconstruction in rabbits. Arthroscopy 26: 968-976, 2010.

15. Dong Y, Zhang Q, Li Y, Jiang J and Chen S: Enhancement of tendon-bone healing for anterior cruciate ligament (ACL) reconstruction using bone marrow-derived mesenchymal stem cells infected with BMP-2. Int J Mol Sci 13: 13605-13620, 2012.

16. Woad KJ, Hunter MG, Mann GE, Laird M, Hammond AJ and Robinson RS: Fibroblast growth factor 2 is a key determinant of vascular sprouting during bovine luteal angiogenesis. Reproduction 143: 35-43, 2012

17. Fei Y, Xiao L, Doetschman T, Coffin DJ and Hurley MM: Fibroblast growth factor 2 stimulation of osteoblast differentiation and bone formation is mediated by modulation of the Wnt signaling pathway. J Biol Chem 286: 40575-40583, 2011.

18. Tsurushima H, Marushima A, Suzuki K, Oyane A, Sogo Y, Nakamura K, Matsumura A and Ito A: Enhanced bone formation using hydroxyapatite ceramic coated with fibroblast growth factor-2. Acta Biomater 6: 2751-2759, 2010.

19. Chen M, Song K, Rao N, Huang M, Huang Z and Cao Y: Roles of exogenously regulated $\mathrm{bFGF}$ expression in angiogenesis and bone regeneration in rat calvarial defects. Int J Mol Med 27: 545-553, 2011

20. Chen B, Qin J, Wang H, Magdalou J and Chen L: Effects of adenovirus-mediated bFGF, IL-1Ra and IGF-1 gene transfer on human osteoarthritic chondrocytes and osteoarthritis in rabbits. Exp Mol Med 42: 684-695, 2010.

21. Hillgenberg $M$, Hofmann C, Stadler $H$ and Löser $P$. High-efficiency system for the construction of adenovirus vectors and its application to the generation of representative adenovirusbased cDNA expression libraries. J Virol 80: 5435-5450, 2006.

22. Mi Z, Ghivizzani SC, Lechman ER, Jaffurs D, Glorioso JC, Evans CH and Robbins PD: Adenovirus-mediated gene transfer of insulin-like growth factor 1 stimulates proteoglycan synthesis in rabbit joints. Arthritis Rheum 43: 2563-2570, 2000

23. Lin HT, Tsai HY, Liu CP and Yuan TT: Comparability of bovine virus titers obtained by TCID $_{50} / \mathrm{ml}$ and FAID50/ml. J Virol Methods 165: 121-124, 2010.

24. Bae SE, Bhang SH, Kim BS and Park K: Self-assembled extracellular macromolecular matrices and their different osteogenic potential with preosteoblasts and rat bone marrow mesenchymal stromal cells. Biomacromolecules 13: 2811-2820, 2012.

25. Deng Y, Li TQ, Yan YE, Magdalou J, Wang H and Chen LB Effect of nicotine on chondrogenic differentiation of rat bone marrow mesenchymal stem cells in alginate bead culture. Biomed Mater Eng 22: 81-87, 2012.
26. Deng Y, Zhou H, Yan C, Wang Y, Xiao C, Gu P and Fan X: In vitro osteogenic induction of bone marrow stromal cells with encapsulated gene-modified bone marrow stromal cells and in vivo implantation for orbital bone repair. Tissue Eng Part A 20: 2019-2029, 2014.

27. Venkatesan J, Bhatnagar I, Manivasagan P, Kang $\mathrm{KH}$ and Kim SK: Alginate composites for bone tissue engineering: A review. Int J Biol Macromol 72: 269-281, 2015.

28. Lee KY and Mooney DJ: Alginate: Properties and biomedical applications. Prog Polym Sci 37: 106-126, 2012.

29. Qiu Y, Wang X, Zhang Y, Carr AJ, Zhu L, Xia Z and Sabokbar A: In vitro two-dimensional and three-dimensional tenocyte culture for tendon tissue engineering. J Tissue Eng Regen Med 10 E216-E226, 2016.

30. Moshaverinia A, Xu X, Chen C, Akiyama K, Snead ML and Shi S: Dental mesenchymal stem cells encapsulated in an alginate hydrogel co-delivery microencapsulation system for cartilage regeneration. Acta Biomater 9: 9343-9350, 2013

31. Caliari SR and Harley BA: Composite growth factor supplementation strategies to enhance tenocyte bioactivity in aligned collagen-GAG scaffolds. Tissue Eng Part A 19: 1100-1112, 2013.

32. Caliari SR, Weisgerber DW, Ramirez MA, Kelkhoff DO and Harley BA: The influence of collagen-glycosaminoglycan scaffold relative density and microstructural anisotropy on tenocyte bioactivity and transcriptomic stability. J Mech Behav Biomed Mater 11: 27-40, 2012.

33. Cai TY, Zhu W, Chen XS, Zhou SY, Jia LS and Sun YQ: Fibroblast growth factor 2 induces mesenchymal stem cells to differentiate into tenocytes through the MAPK pathway. Mol Med Rep 8: 1323-1328, 2013.

34. Qiu Y, Wang X, Zhang Y, Carr AJ, Zhu L, Xia Z and Sabokbar A: Development of a refined tenocyte expansion culture technique for tendon tissue engineering. J Tissue Eng Regen Med 8: 955-962, 2014

35. Song X, Liu S, Qu X, Hu Y, Zhang X, Wang T and Wei F: BMP2 and VEGF promote angiogenesis but retard terminal differentiation of osteoblasts in bone regeneration by up-regulating Id1 Acta Biochim Biophys Sin (Shanghai) 43: 796-804, 2011.

36. Park SY, Kim KH, Shin SY, Koo KT, Lee YM and Seol YJ: Dual delivery of rhPDGF-BB and bone marrow mesenchymal stromal cells expressing the BMP2 gene enhance bone formation in a critical-sized defect model. Tissue Eng Part A 19: 2495-2505, 2013.

37. Murray SJ, Santangelo KS and Bertone AL: Evaluation of early cellular influences of bone morphogenetic proteins 12 and 2 on equine superficial digital flexor tenocytes and bone marrowderived mesenchymal stem cells in vitro. Am J Vet Res 71: 103-114, 2010.

38. Salingcarnboriboon R, Yoshitake H, Tsuji K, Obinata M, Amagasa T, Nifuji A and Noda M: Establishment of tendonderived cell lines exhibiting pluripotent mesenchymal stem cell-like property. Exp Cell Res 287: 289-300, 2003

39. Steinert AF, Kunz M, Prager P, Barthel T, Jakob F, Nöth U, Murray MM, Evans CH and Porter RM: Mesenchymal stem cell characteristics of human anterior cruciate ligament outgrowth cells. Tissue Eng Part A 17: 1375-1388, 2011.

40. Hashimoto Y, Naka Y, Fukunaga K, Nakamura H and Takaoka K: ACL reconstruction using bone-tendon-bone graft engineered from the semitendinosus tendon by injection of recombinant BMP-2 in a rabbit model. J Orthop Res 29: 1923-1930, 2011.

41. Martinek V, Latterman C, Usas A, Abramowitch S, Woo SL, Fu FH and Huard J: Enhancement of tendon-bone integration of anterior cruciate ligament grafts with bone morphogenetic protein-2 gene transfer: A histological and biomechanical study. J Bone Joint Surg Am 84-A: 1123-1131, 2002

42. Hou Y, Mao Z, Wei X, Lin L, Chen L, Wang H, Fu X, Zhang J and Yu C: Effects of transforming growth factor-betal and vascular endothelial growth factor 165 gene transfer on Achilles tendon healing. Matrix Biol 28: 324-335, 2009.

43. Wang L, Zou D, Zhang S, Zhao J, Pan K and Huang Y: Repair of bone defects around dental implants with bone morphogenetic protein/fibroblast growth factor-loaded porous calcium phosphate cement: A pilot study in a canine model. Clin Oral Implants Res 22: 173-181, 2011. 